\title{
Original
}

\section{Primera nefrectomia transvaginal híbrida por cáncer renal}

\author{
María J. Ribal Caparrós, Lluís Peri Cusí, Alejandro Molina Cabeza, Alexa García Larrosa, \\ Francisco Carmona, Antonio Alcaraz Asensio
}

Departamento de Urología. Hospital Clínic. Universitat de Barcelona, España.

\section{Resumen}

La cirugía NOTES (Natural Orifice Transluminal Endoscopic Surgery) es una modalidad quirúrgica que utiliza las vísceras huecas para acceder a la cavidad peritoneal, evitando así incisiones en la piel. Si combinamos este tipo de cirugía con la laparoscopia convencional, obtenemos una técnica híbrida que nos permitirá abordar órganos grandes, como el riñón, disminuyendo al máximo las incisiones cutáneas. Presentamos el primer caso de nefrectomía radical híbrida transvaginal por cáncer renal. Para poder realizar este procedimiento, se utilizó el acceso vaginal para la colocación de una cámara deflectable y la asistencia de dos puertos abdominales. La incisión del trócar vaginal se amplió para la extracción de la pieza quirúrgica. La nefrectomía híbrida transvaginal NOTES asistida debe considerarse, una técnica quirúrgica factible y reproducible que debe ser considerada como opción terapéutica en pacientes mujeres candidatas a nefrectomía por cáncer renal.

Palabras clave: Nefrectomía radical. NOTES híbrida. Transvaginal.

\section{First report on hybrid transvaginal nephrectomy for renal cancer}

\section{Abstract}

NOTES (Natural Orifice Transluminal Endoscopic Surgery) is a surgical modality that uses empty organs as an access to the peritoneal cavity, avoiding skin incisions. If we combine this surgery with the classical laparoscopic approach, a new hybrid technique is obtained. This approach will permit us to work on wide operating fields with large organs, as the kidney, minimizing skin incisions. We present the first hybrid transvaginal radical nephrectomy due to renal cancer. In order to perform this procedure, we used a vaginal access for introduction of a deflectable camera and the assistance of two additional abdominal trocars. The vaginal incision for the trocar was enlarged for organ removal. Hybrid transvaginally NOTES assisted radical nephrectomy is a reproducible and feasible technique that has to be kept in mind for women who are candidates to nephrectomy for renal cancer.

Keywords: Radical nephrectomy. Hybrid NOTES. Transvaginal.

La cirugía endoscópica a través de orificios naturales, conocida como NOTES (Natural Orifice Transluminal Endoscopic Surgery) es una modalidad quirúrgica emergente que utiliza instrumentos endoscópicos a través de vísceras huecas para acceder a la cavidad peritoneal y así permitir actos quirúrgicos sin necesidad de incisiones. Es posible combinar la técnica de NOTES con la laparoscopia convencional resultando en una técnica híbrida en la que tampoco se realizan incisiones para la extracción de la pieza. Así se publicó la nefrectomía híbrida transvaginal por riñón atrófico y nosotros presentamos la primera nefrectomía híbrida transvaginal por cáncer renal realizada en nuestro Departamento de Urología, del Hospital Clínic de Barcelona en marzo de 2008.

\section{CASO CLÍnICO}

Paciente mujer de 65 años de edad remitida a nuestro centro con el diagnóstico de tumor renal derecho. Tras un episodio de dolor en flanco derecho acudió a médico de cabecera que inició estudio. Se realizó ecografía abdominal que evidenció una tumoración sólida en el polo superior del riñón derecho. Se procedió a la confirmación diagnóstica mediante TAC abdominal que informó de la presencia de tumoración en polo superior del riñón derecho de $5 \mathrm{~cm}$. de diámetro y captación heterogénea de contraste. Con la orientación de tumor renal estadio Tlb la paciente fue informada de la necesidad de someterse a nefrectomía radical. Bajo la aprobación del Comité Ético de nuestro hospital la paciente fue informada de la posibilidad de some- 
terse a una nueva técnica quirúrgica: nefrectomía transvaginal en el contexto del programa NOTES de nuestro departamento.

\section{Técnica quirúrgica}

Bajo anestesia general la paciente se colocó en decúbito lateral izquierdo y las piernas en posición de litotomía moderada para permitir el acceso vaginal (Fig. 1). El pneumoperitoneo se consiguió a través de un trócar de $12 \mathrm{~mm}$ colocado en flanco derecho a unos $4 \mathrm{~cm}$ del ombligo. La presión intrabdominal se mantuvo en todo momento a $12 \mathrm{mmHg}$. Se introdujo en la cavidad peritoneal, una óptica de laparoscopia convencional de $0^{\circ}$ y bajo visión directa se colocó un puerto adicional en flanco derecho de $5 \mathrm{~mm}$. Mediante una pinza se procedió a la retracción del útero y exposición del fondo de saco vaginal. Bajo visión directa se colocó un trócar especial de cirugía bariátrica de $12 \mathrm{~mm}$ a través de la vagina perforando el fondo de saco vaginal. Se retiró la óptica convencional y a través del trócar vaginal se introdujo una óptica de $0^{\circ}$ con el extremo flexible que fue la que se utilizó para asistir el procedimiento (Fig. 2).

La técnica se llevó a cabo siguiendo los principios de la nefrectomía laparoscópica convencional si bien con una visión más caudal de la que normalmente se obtiene dado el especial emplazamiento de la cámara. Se incidió la línea a de Toldt y se movilizó el colon derecho hasta identificar el músculo psoas. Se identificó y disecó el uréter derecho y se seccionó mediante Ligasure. Se alcanzó el hilio renal tras liberación del polo inferior del riñón derecho. Se trataba de un pedículo único. La arteria se disecó y ligó entre clips tipo

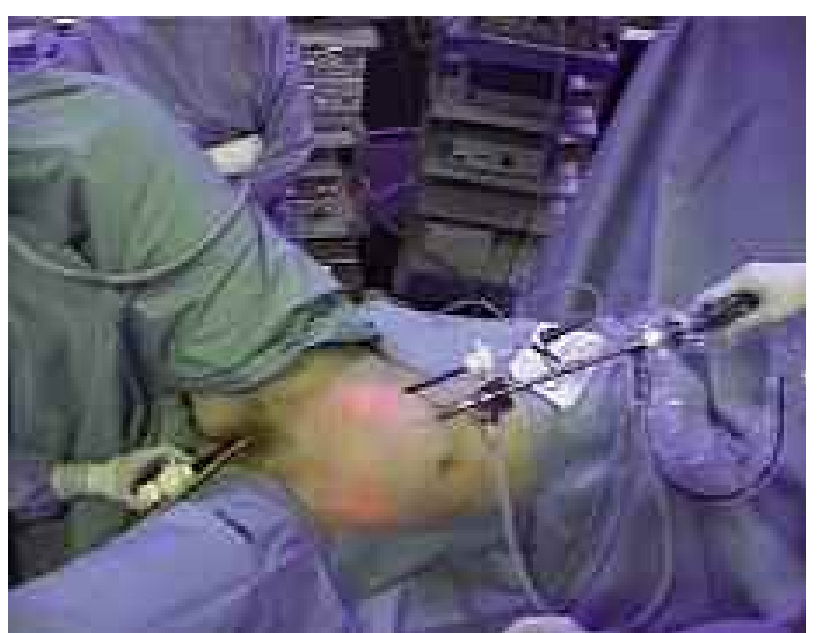

FIGURA 1. Colocación de la paciente en decúbito lateral izquierdo y las piernas en posición de litotomía moderada.

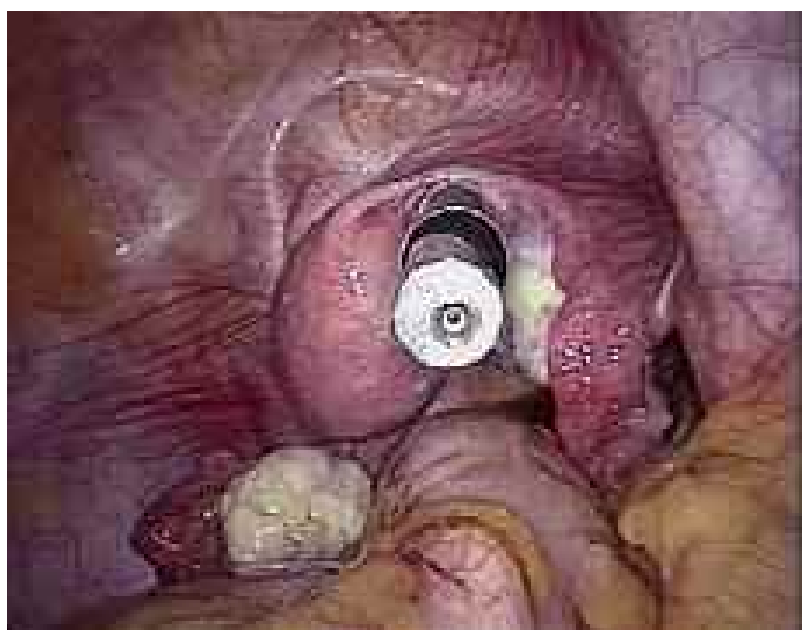

FIGURA 2. Entrada de la cámara flexible a través del trócar colocado en el fondo de saco vaginal posterior.

Hemo-o-lock, realizándose el mismo procedimiento con la vena renal derecha. Se liberó la cara posterior del riñón y el polo superior preservando la glándula suprarrenal. Se embolsó la pieza de nefrectomía radical derecha a través de endobag introducido por el trócar vaginal y bajo visión directa, asistida por cámara laparoscópica convencional a través del trócar abdominal, se procedió a la exteriorización del espécimen quirúrgico a través de la vagina. La colpotomía fue suturada con puntos sueltos de Vycril 2/0. El procedimiento se realizó en un tiempo total de 103 minutos con un sangrado total de $200 \mathrm{ml}$. El postoperatorio fue correcto y la paciente fue dada de alta a las 72 horas. Desde el momento del alta la paciente inició sus actividades habituales. La anatomía patológica definitiva informó de carcinoma de células renales (células claras) estadio pTlb Furhmann II.

\section{DISCUSIÓN}

La Urología como especialidad puede considerarse pionera en el desarrollo de la cirugía endoscópica, o a través de orificios naturales. De hecho la resección transuretral es un procedimiento plenamente establecido en nuestro armamento terapéutico. De esta forma podemos pensar que la Urología debe mantener su papel de abanderada en el desarrollo de las nuevas técnicas quirúrgicas englobadas bajo la denominación de NOTES.

De hecho una de las primeras publicaciones sobre aplicación de NOTES en modelo animal, se trata de una serie de nefrectomía transvaginal en modelo porcino, publicada por Gettman et al. en 2002. En cinco de los 6 procedimientos realizados 
en su serie se procedió a la cirugía asistida por trócar abdominal de $5 \mathrm{~mm}$. Sólo en un caso el procedimiento se realizó íntegramente por vía vaginal ${ }^{1}$.

El desarrollo de modelos animales permitió el avance en la investigación de nuevas técnicas quirúrgicas sin incisión, y en 2004 Kallo et al. publican una serie en modelo porcino de peritoneoscopia transgástrica. De hecho esta publicación es considerada la primera referencia a la técnica de NOTES tal y como hoy la consideramos ${ }^{2}$. A partir de ese momento se inicia la publicación creciente de procedimientos realizados vía transgástrica, incluyendo ligadura de trompa de Falopio $^{3}$, colecistectomía ${ }^{4}$, gastroyeyunostomía ${ }^{5}$ y esplenectomía ${ }^{6}$. No es hasta 2004, que en el Congreso de la Sociedad de Endoscopia Gastrointestinal de la India se publica el primer caso de cirugía combinada en humanos, concretamente una apendicectomía transgástrica ${ }^{7}$. En 2007 se publica la primera cirugía NOTES pura en humanos por el grupo del IRCAD-EITS (Institut de Recherche contre les Cancers de 1'Appareil Digestif (Institut of Digestive Cancer Research)Eurpoean Institut of Telesurgery). Concretamente se publica la primera colecistectomía transvaginal en una paciente mujer de 30 años afecta de colelitiasis sintomática. Para crear el pneumoperitoneo se utilizó un puerto de $2 \mathrm{~mm}$ abdominal que durante el procedimiento se utilizó para la retracción de la vesícula. El postoperatorio fue correcto y la paciente fue dada de alta en 48 horas $^{8}$.

Las ventajas ya conocidas de la laparoscopia sobre la cirugía abierta llevan a los defensores de la cirugía mínimamente invasiva a considerar que supondrá ventajas sobre la laparoscopia. Las justificaciones para NOTES estriban en la ausencia de cicatrices, la mejora de la cosmética, la reducción del disconfort y el dolor postoperatorio, la reducción de posibilidad de hernia postoperatoria, reducir la posibilidad de bridas, y en definitiva reducir el trauma derivado de una cirugía efectiva. La eliminación de la cicatriz supone también eliminar cualquier aspecto visible que pueda recordar o hacer patente la cirugía en $\mathrm{si}^{9}$.

$\mathrm{El}$ abordaje transvaginal parece acomodarse perfectamente a los deseos de mejora cosmética y ausencia de cicatriz que se persiguen con el desarrollo de NOTES, así como permite la extracción de piezas quirúrgicas de tamaño considerable, lo que la hace especialmente atractiva como vía de abordaje en la cirugía ablativa, incluso oncológica.
En 2002, a la vez que Gettmann desarrollaba el modelo porcino de nefrectomía transvaginal, Gill et al. publicaban su primera serie de nefrectomía laparoscópica con extracción vaginal de la pieza. Concretamente 10 mujeres con media de edad de 67 años fueron sometidas a nefrectomía radical laparoscópica por tumor renal y posterior colpotomía para extraer la pieza. Los autores señalan en el artículo que tal abordaje para la extracción se ha convertido en el estándar para mujeres sometidas a cirugía ablativa renal ${ }^{10}$.

No es hasta 2008 que se publica la primera nefrectomía transvaginal hibrida por atrofia renal y riñón no funcionante por el grupo de Brasil de Branco et al. A diferencia de la serie de Gill, no se trata de una nefrectomía laparoscópica convencional, sino que el procedimiento se realiza enteramente a través de la vagina, asistido por dos puertos abdominales únicamente, que permiten la visualización y la ayuda para retracción del riñón ${ }^{11}$. En nuestro caso también procedimos a una técnica híbrida, aunque a diferencia del grupo brasileño, el acceso vaginal lo utilizamos para la visión gracias a una cámara de punta flexible (Olympus). Ello permite trabajar con dos únicos puertos abdominales que aunque en posición muy caudal, para adecuarse a la visión caudal de la cámara, permiten la realización de la nefrectomía de forma muy similar a la nefrectomía laparoscópica convencional. Además permite la utilización de material de laparoscopia convencional, obviando los problemas del material endoscópico diseñado para NOTES puro que impiden la correcta retracción del órgano y presenta limitaciones para su uso dentro de la cavidad peritoneal. De hecho, el material diseñado para NOTES ha sido criticado en determinados momentos y para determinados procedimientos precisamente por sus notables limitaciones para la retracción, el reducido tamaño de los canales de trabajo que no permiten utilizar elementos de ligadura potentes, y la incongruencia espacial del material demasiado flexible para utilizarse libre en la cavidad peritoneal. De hecho ya se ha iniciado una corriente que defiende el abordaje híbrido mínimamente invasivo sobre el NOTES puro sin asistencia laparoscópica. Las ventajas que se le suponen al abordaje híbrido es que permite la perforación de la luz del órgano, a través del cual trabajar, bajo visión directa minimizando los riesgos de daño de órganos adyacentes. Además mejora de forma importante la orientación del material endoscópico en la cavidad peritoneal. Permite la retracción de los órganos a 
disecar de una forma efectiva cosa que la técnica puro no permite por la flexibilidad de los instrumentos. En definitiva la técnica híbrida permite mejorar la seguridad del procedimiento NOTES puro mientras que minimiza la agresividad de la laparoscopia convencional ${ }^{12}$.

Actualmente la cirugía sin cicatrices se halla en pleno desarrollo y asistimos a la creciente publicación de procedimientos diversos en el marco de la minimización de la agresión. Así actualmente se está desarrollando la conocida como cirugía a través de puerto único. A través de un único puerto abdominal con diferentes canales de trabajo se realizan procedimientos laparoscópicos convencionales. Los modelos animales se están utilizando para la mejora de la técnica y la expansión de indicaciones. Así se ha publicado la nefrectomía a través de puerto único con un sistema magnético de guía de material en modelo porcino $^{13}$. El campo de la cirugía mínimamente invasiva se halla en plena expansión, siendo la Urología una de las áreas en las que se está viviendo una expansión importante. De esta forma ya tenemos trabajos que perfeccionan y buscan nuevas indicaciones y modalidades de la cirugía sin cicatriz, como la crioablación renal a través de NOTES en modelo porci$\mathrm{no}^{14}$, o la nefrectomía transvaginal con puerto único en modelo porcino tambié ${ }^{15}$, o la nefrectomía por NOTES asistida por $\operatorname{robot}^{16}$, o la que se ha venido a llamar tercera generación de NOTES presentando una serie de nefrectomía combinada transvesical y transgástrica en un modelo porcino ${ }^{17}$.

La cirugía mínimamente invasiva o cirugía sin cicatrices supone una demanda técnica importante que debemos enfrentar y procurar su desarrollo porque supone un paso adelante al conseguido en su momento por la cirugía laparoscópica.

\section{CONCLUSIONES}

La nefrectomía transvaginal es una técnica quirúrgica factible y reproducible incluida dentro del concepto NOTES que debe ser considerada como una opción terapéutica válida en pacientes mujeres que deban ser sometidas a nefrectomía por tumor renal. Es fundamental la correcta selección de las pacientes a tratar para garantizar la seguridad del procedimiento. Es necesario el seguimiento a largo plazo para la estandarización de la técnica.

\section{REFERENCIAS}

1. Gettman MT, Lotan Y, Napper CA, et al. Transvaginal laparoscopic nephrectomy: development and feasibility in the porcine model. Urology. 2002;59(3):446-450.

2. Kalloo AN, Singh VK, Jagannath SB, et al. Flexible transgastric peritoneoscopy: a novel approach to diagnostic and therapeutic interventions in the peritoneal cavity. Gastrointest Endosc. 2004;60(1):114-117.

3. Jagannath SB, Kantsevoy SV, Vaughn CA. et al. Peroral transgastric endoscopic ligation of fallopian tubes with long-term survival in a porcine model. Gastrointest Endosc. 2005;61(3): 449-453.

4. Park PO, Bergstrom M, Ikeda K. et al. Experimental studies of transgastric gallbladder surgery: cholecystectomy and cholecystogastric anastomosis (videos). Gastrointest Endosc. 2004;61(4):601-606.

5. Kantsevoy SV, Jagannath SB, Niiyama H. et al. Endoscopic gastrojejunostomy with survival in a porcine model. Gastrointest Endosc. 2005;62(2):287-292.

6. Kantsevoy SV, Hu B, Jagannath SB. et al. Transgastric endoscopic splenectomy: is it possible? Surg Endosc. 2006;20(3): 522-525.

7. Reddy DN, Rao GV. Transgastric approach to the peritoneal cavity: are we on the right track? Gastrointest Endosc. 2007; 65(3):501-502.

8. Marescaux J, Dallemagne B, Perretta S. et al. Surgery without scars: report of transluminal cholecystectomy in a human being. Arch Surg. 2007;142(9):823-826.

9. Swain P. Nephrectomy and natural orifice translumenal endoscopy (NOTES): transvaginal, transgastric, transrectal, and transvesical approaches. J Endourol. 2008;22(4):811-818.

10. Gill IS, Cherullo EE, Meraney AM. et al. Vaginal extraction of the intact specimen following laparoscopic radical nephrectomy. J Urol. 2002;167(1):238-241.

11. Branco AW, Branco Filho AJ, Kondo W. et al. Hybrid transvaginal nephrectomy. Eur Urol. 2008;53(6):1290-1294.

12. Shih SP, Kantsevoy SV, Kalloo AN. et al. Hybrid minimally invasive surgery a bridge between laparoscopic and translumenal surgery. Surg Endosc. 2007;21(8):1450-1453.

13. Zeltser IS, Bergs R, Fernandez R. et al. Single trocar laparoscopic nephrectomy using magnetic anchoring and guidance system in the porcine model. J Urol. 2007;178(1):288-191.

14. Crouzet S, Haber GP, Kamoi K. et al. Natural orifice translumenal endoscopic surgery (NOTES) renal cryoablation in a porcine model. BJU Int. 2008;102(11):1715-1718.

15. Clayman RV, Box GN, Abraham JB. et al. Rapid communication: transvaginal single-port NOTES nephrectomy: initial laboratory experience. J Endourol. 2007;21(6):640-644.

16. Box GN, Lee HJ, Santos RJ. et al. Rapid communication: robotassisted NOTES nephrectomy: initial report. J Endourol. 2008; 22(3):503-506.

17. Lima E, Rolanda C, Pego JM. et al. Third-generation nephrectomy by natural orifice transluminal endoscopic surgery. J Urol. 2007;178(6):2648-2654.

Correspondencia autor: Dra. María J. Ribal Caparrós Departamento de Urología. Hospital Clínic i Provincial Villarroel 170-08036. Barcelona. Tel. 932275545

E-mail autor: mjribal@clinic.ub.es.

Información artículo: Original - Laparoscopia

Trabajo recibido: enero 2009

Trabajo aceptado: febrero 2009 